\title{
3D Syllabus: Interactive Visualization of Indexes to Multimedia Training Content
}

\author{
Kyuman Song ${ }^{1}$, Surapong Lertsithichai ${ }^{2}$, and Patrick Chiu ${ }^{3}$ \\ ${ }^{1}$ General Dynamics C4 Systems, 2100 Wharton st. Pittsburgh, PA 15203 USA \\ ksong@post.harvard.edu \\ ${ }^{2}$ Faculty of Architecture, Silpakorn University, Bangkok, Thailand \\ surapong@post . harvard. edu \\ ${ }^{3}$ FX Palo Alto Laboaratory, 3400 Hillview Ave., Bldg. 4, Palo Alto, CA 94304 USA \\ chiu@ fxpal.com
}

\begin{abstract}
Indexes such as bookmarks and recommendations are helpful for accessing multimedia documents. This paper describes the 3D Syllabus system, which is designed to visualize indexes to multimedia training content along with the information structures. A double-sided landscape with balloons and cubes represents the personal and group indexes, respectively. The $2 \mathrm{D}$ ground plane organizes the indexes as a table and the third dimension of height indicates their importance scores. Additional visual properties of the balloons and cubes provide other information about the indexes and their content. Paths are represented by pipes connecting the balloons. A preliminary evaluation of the 3D Syllabus prototype suggests that it is more efficient than a typical training CD-ROM and is more enjoyable to use.
\end{abstract}

\section{Introduction}

Digital multimedia documents are becoming more widely available with rapid advances in technologies for content creation and distribution. For accessing and browsing documents in a collection, indexes are helpful and often indispensable. Examples of indexes are bookmarks, annotations, recommendations by other users, and sequences prescribed by an instructor.

This paper describes techniques for 3D visualization and interaction with indexes to multimedia training documents. These techniques were developed in response to a multimedia system used to train technicians in the maintenance and repair of printers and copiers from the Fuji-Xerox Company. This multimedia content is distributed on a CD-ROM and produced with Macromedia Authorware. The user interface has hyperlinks and buttons for page turning. The content is organized hierarchically. At the top level are modules, and within each module is a tree diagram (Fig. 1-A and B). The leaves of a tree diagram are pages that can be accessed and viewed. The pages contain text, images, and video clips (Fig. 1-C).

There are several problems with this visualization and user interface, which also occur with typical hyperlinked training CD-ROMs. For the training application in Fig. 1, the complicated hierarchical structure is time-consuming to navigate. Limited 
information is displayed; the tree view for each module shows the structure, but the screen does not have room to show all the trees. Learning paths are inflexible because they are determined by the tree structures, and instructors and users cannot create customized paths. The interactions restrict the user to clicking on hyperlinks for navigation and page turning. Knowledge sharing between students is not supported.

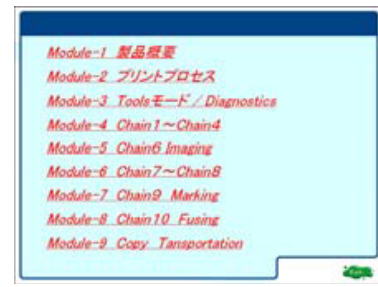

A. Modules

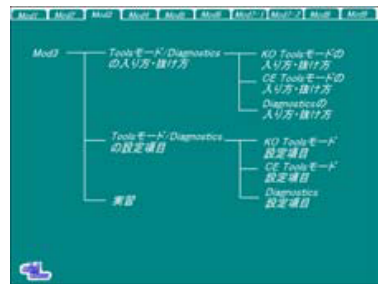

B. Trees

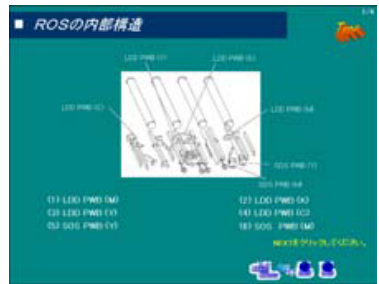

C. Typical contents

Fig. 1. Screen shots of the Fuji-Xerox Training CD-ROM

The 3D techniques presented in this paper address these problems. An overview of the entire content structure and relationships can be displayed. A property of 3D space is that it is scalable and essentially infinite. Consequently, through 3D navigation, the user can vary the viewpoint to obtain better views of the entire content or certain parts of the content. Paths are better supported in 3D than in 2D, since paths that crisscross or pass over nodes cause difficulties in a $2 \mathrm{D}$ visualization. For the specific problem of showing personal and group indexes, we have designed a double-sided 3D landscape.

Other visualizations for multimedia or hyperlinked contents are surveyed in [1]. None of these utilizes a double-sided landscape. Alternatively, a simple listing of multimedia data (e.g. [2]) is easy to use but does not provide a view of the information structures. Context maps like the Learning Landscape [3] provide different views (by topic, faculty, people, places); however, distortion and instability are drawbacks with this type of mapping.

\section{3D Syllabus}

A screen shot of the 3D Syllabus is shown in Fig. 2. The content visualized is taken from the Fuji-Xerox training CD-ROM. The content set is organized as a table that is represented by a grid of square tiles (see Fig. 3). These tiles are set on the flat ground plane in the 3D landscape. Spaces between the tiles allow the user to see the objects above and below ground. Part names are listed along one axis and task types are listed along the other axis.

A balloon (or sphere) represents an index to the multimedia content; a balloon has a string connecting it to the square tile beneath it. Clicking on a balloon accesses or plays the content corresponding to the square (i.e. one of the content pages in Fig. 1C). A balloon's size indicates the amount of content for that lesson; the size can be computed from the number of pages or the file size. The color patches on a balloon indicate the number and type of media. After content has been accessed, the user can 
give it a rating by directly manipulating the balloon's height (or through a dialog box). The height of a balloon indicates the rating: very low, low, medium, high, very high.

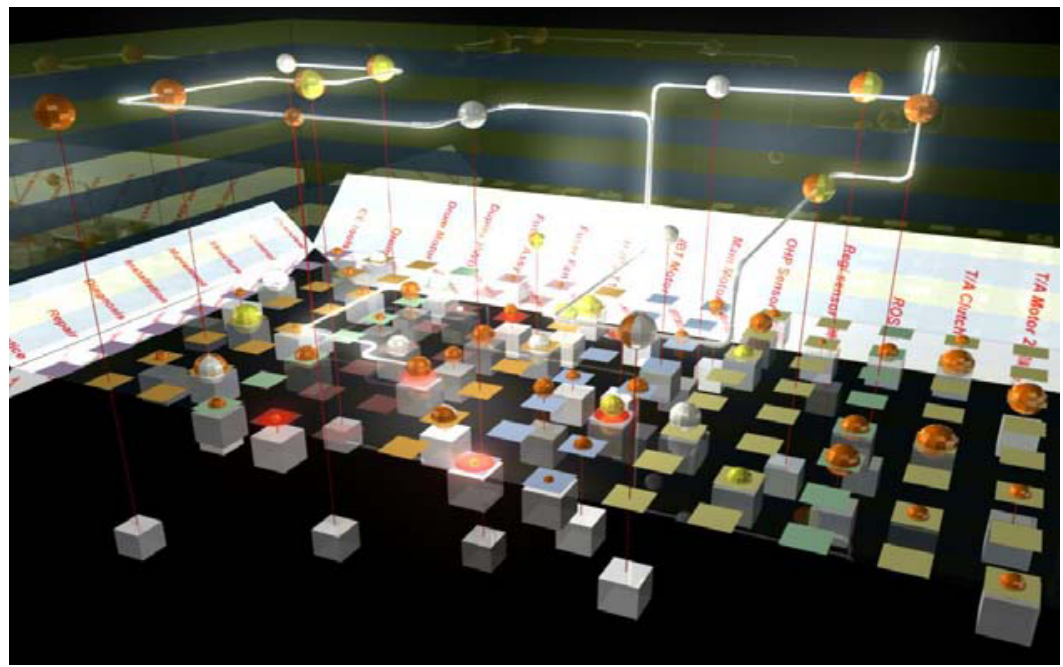

Fig. 2. 3D Syllabus

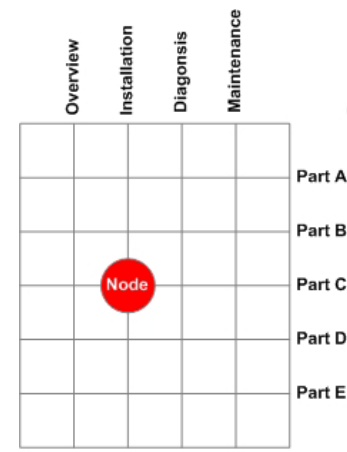

Square Tiles (top view)

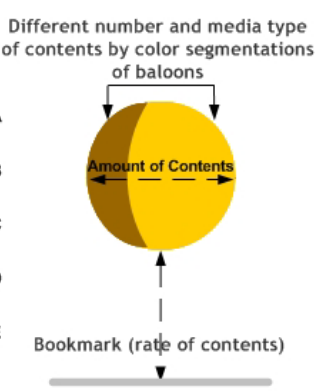

Balloon (side view)

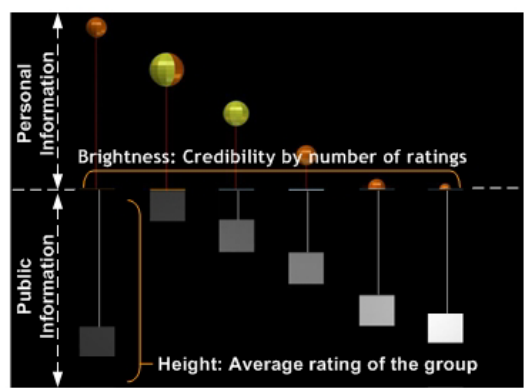

Double-sided landscape (side view)

Fig. 3. Concept diagram of 3D Syllabus

It is sometimes useful to know whether a balloon has been visited and its content viewed. A visited balloon has a red circle painted on its square tile (shown in Fig. 2). Color is used to visually group tiles of related content. For example, tiles of the same color belong to the same training module. When the cursor hovers over a balloon or tile, tooltips can be used to provide text information about the title, content size, height, tile group, etc. The pipes connecting the balloons represent paths through the content. For example, an instructor can prescribe a course by using a path. 
Alternatively, a path can show the history of the lessons taken by a user studying independently. Arrows on the pipes can indicate direction (not shown in figure).

The visualization is a double-sided landscape with one side for personal indexes and the other side for group indexes. See Fig. 2 and 3. Examples are bookmarks (personal index) and recommendations by other students (group index). The side with the balloons is the personal side, and the side with the cubes is the group side. For the training application, the group can comprise the students in the class. The height of a cube is given by the average rating of the group. The brightness indicates how many people rated the content; this is important to know since an average rating from a small number of people may be less reliable. Clicking on a cube accesses group content such as the annotations created by other people.

\section{Prototype Evaluation}

A prototype of this system built in Macromedia Director has been tested in comparison with the existing system by five people. After a five-minute introduction, users were asked to find five particular topics in both systems. For the first given task, users spent 5-20 seconds finding the topic in the 3D syllabus compared to 60-500 seconds using the current system. After the test, users were asked to draw a mental map of the location of specific information. Significantly, users recalled the location of contents more precisely from their mental map of the 3D grid system. On the other hand, users couldn't remember the location of information on more than two topics from the existing system. Another benefit of 3D has to do with the user experience. From our informal user testing of an early prototype, users commented that $3 \mathrm{D}$ interfaces are more enjoyable to use. While the response to the 3D Syllabus has been encouraging, further testing with real users at Fuji-Xerox will be needed to ascertain the usefulness of the system.

Acknowledgements. We thank Laurent Denoue, Tohru Fuse, and Lynn Wilcox for their help and comments.

\section{References}

1. Benford, S., Taylor, I., Brailsford, D., Koleva, B., Craven, M., Fraser, M., Reynard G. Greenhalgh, C. Three dimensional visualization of the World Wide Web. ACM Computing Surveys 31(4), Dec. 1999.

2. Girgensohn, A., Boreczky, J., Wilcox, L., Foote, J. Facilitating video access by visualizing automatic analysis. Proceedings of Interact '99, pp. 205-212.

3. Learning Landscape, ETH (Federal Institute of Technology Zurich), http://www.dgj.ch/ ethworld 